\title{
PRESERVATION OF SOME PARTIAL ORDERINGS UNDER POISSON SHOCK MODELS
}

\author{
HARSHINDER SINGH AND \\ KANCHAN JAIN, Panjab University
}

\begin{abstract}
Suppose each of the two devices is subjected to shocks occurring randomly as events in a Poisson process with constant intensity $\lambda$. Let $\bar{P}_{k}$ denote the probability that the first device will survive the first $k$ shocks and let $\bar{Q}_{k}$ denote such a probability for the second device. Let $\bar{F}(t)$ and $\bar{G}(t)$ denote the survival functions of the first and the second device respectively. In this note we show that some partial orderings, namely likelihood ratio ordering, failure rate ordering, stochastic ordering, variable ordering and mean residual-life ordering between the shock survival probabilities $\bar{P}_{k}$ and $\bar{Q}_{k}$ are preserved by the corresponding survival functions $\bar{F}$ and $\bar{G}$.
\end{abstract}

RELIABILITY; POSITIVE AGEING ClASSES OF DISTRIBUTIONS; TOTAL POSITIVITY; VARIATION DIMINISHING POLICY

\section{Introduction}

Suppose that a device is subjected to shocks occurring randomly as events in a Poisson process with constant intensity $\lambda$. Suppose further that the device has probability $\bar{P}_{k}$ of surviving the first $k$ shocks, where $1=P_{0} \geqq \bar{P}_{1} \geqq \cdots$. The survival function of the device is given by

$$
\bar{F}(t)=\sum_{k=0}^{\infty} \frac{\exp (-\lambda t)(\lambda t)^{k}}{k !} \bar{P}_{k} .
$$

Esary et al. (1973) have shown that the positive ageing classes of life distributions, namely a $\mathrm{PF}_{2}$ density, increasing failure rate (IFR), decreasing mean residual life (DMRL), increasing failure rate average (IFRA), new better than used (NBU) and new better than used in expectation (NBUE) are preserved under the transformation (1.1) in the sense that if the shock survival probability $\bar{P}_{k}$ belongs to a discrete version of one of the above classes, then the continuous-time survival probability $\bar{F}(t)$ belongs to that class. Klefsjö (1981) has established that if $\bar{P}_{k}$ belongs to the discrete version of the harmonic new better than used in expectation (HNBUE) class of life distributions, than $\bar{F}(t)$ belongs to the HNBUE class.

Consider another device which is also subjected to shocks occurring randomly as events in a Poisson process with same constant intensity $\lambda$ and the device has probability $\bar{Q}_{k}$ of surviving the first $k$ shocks, where $1=\bar{Q}_{1} \geqq \bar{Q}_{1} \geqq \cdots$. The survival function of this device is given by

$$
\bar{G}(t)=\sum_{k=0}^{\infty} \frac{\exp (-\lambda t)(\lambda t)^{k}}{k !} \bar{Q}_{k} .
$$

In Section 2 of this note, we show that partial orderings, namely likelihood ratio (LR) ordering, failure rate (FR) ordering, stochastic (St) ordering, variable (V) ordering and mean

Received 20 December 1988.

Postal address for both authors: Department of Statistics, Panjab University, Chandigarh-160014, India. 
residual (MR) ordering between the two shock survival probabilities $\bar{P}_{k}$ and $\bar{Q}_{k}$ are preserved by the corresponding survival functions $\bar{F}$ and $\bar{G}$ of the devices.

For definition of these partial orderings, the reader is referred to Ross (1983), Singh (1988), Deshpande et al. (1988).

\section{Preservation of partial orderings}

Let $p_{k}=\bar{P}_{k-1}-\bar{P}_{k}$ and $q_{k}=\bar{Q}_{k-1}-\bar{Q}_{k}, k=1,2,3, \cdots$. We shall denote by $f(t)$ and $g(t)$ the probability density functions corresponding to the survival functions $\bar{F}$ and $\bar{G}$ respectively. These densities are given by

$$
f(t)=\sum_{k=0}^{\infty} \frac{\exp (-\lambda t)(\lambda t)^{k}}{k !} \lambda p_{k+1}
$$

and

$$
g(t)=\sum_{k=0}^{\infty} \frac{\exp (-\lambda t)(\lambda t)^{k}}{k !} \lambda q_{k+1} .
$$

In the derivation of the results, we use the methods of total positivity and in particular the variation diminishing property of the totally positive (TP) function

$$
Z_{k}(t)=\frac{\exp (-\lambda t)(\lambda t)^{k}}{k !}, \quad t \geqq 0, \quad k=0,1,2, \cdots .
$$

(See Karlin (1968), p. 21.)

The results of the note are summarized as follows.

Theorem 2.1.

(i) Let $\bar{P}_{k} \geqq{ }^{\mathrm{LR}} \bar{Q}_{k}$ i.e. let $p_{k+1} / q_{k+1}$ be a non-decreasing function of $k=0,1,2, \cdots$, then $\bar{F} \geqq{ }^{\mathrm{LR}} \bar{G}$ i.e. $f(t) / g(t)$ is a non-decreasing function of $t$.

(ii) Let $\bar{P}_{k} \geqq{ }^{\mathrm{FR}} \bar{Q}_{k}$ i.e.

$$
\frac{p_{k}}{\bar{P}_{k}} \leqq \frac{q_{k}^{-}}{\bar{Q}_{k}}, \quad k=1,2, \cdots
$$

then $\bar{F} \geqq{ }^{\text {FR }} \bar{G}$ i.e.

$$
\frac{f(t)}{\bar{F}(t)} \leqq \frac{g(t)}{\bar{G}(t)} \quad \forall t \geqq 0 .
$$

(iii) Let $\bar{P}_{k} \geqq^{\text {st }} \bar{Q}_{k}$ i.e. $\bar{P}_{k} \geqq \bar{Q}_{k} \forall k=1,2, \cdots$, then $\bar{F} \geqq$ st $\bar{G}$ i.e. $\bar{F}(t) \geqq \bar{G}(t) \forall t \geqq 0$.

(iv) Let $\bar{P}_{k} \geqq^{\mathrm{v}} \bar{Q}_{k}$ i.e.

$$
\sum_{k=j}^{\infty} \bar{P}_{k} \geqq \sum_{k=j}^{\infty} \bar{Q}_{k}, \quad \forall j=0,1,2, \cdots,
$$

then $\bar{F} \geqq^{\mathrm{v}} \bar{G}$ i.e.

$$
\int_{t}^{\infty} \bar{F}(u) d u \geqq \int_{t}^{\infty} \bar{G}(u) d u \quad \forall t \geqq 0 .
$$

(v) Let $\bar{P}_{k} \geqq^{\mathrm{MR}} \bar{Q}_{k}$ i.e. let

$$
\frac{1}{\bar{P}_{j}} \sum_{k=j}^{\infty} \bar{P}_{k} \geqq \frac{1}{\bar{Q}_{j}} \sum_{k=j}^{\infty} \bar{Q}_{k}, \quad j=0,1,2, \cdots,
$$

then $\bar{F} \geqq{ }^{\mathrm{MR}} \bar{G}$ i.e.

$$
\frac{1}{\bar{F}(t)} \int_{t}^{\infty} \bar{F}(u) d u \geqq \frac{1}{\bar{G}(t)} \int_{t}^{\infty} \bar{G}(u) d u \quad \forall t \geqq 0 .
$$


Proof. (i) For any real $c$,

$$
f(t)-c g(t)=\sum_{k=0}^{\infty} \frac{\exp (-\lambda t)(\lambda t)^{k}}{k !}\left(p_{k+1}-c q_{k+1}\right) .
$$

Since $p_{k+1} / q_{k+1}$ is a non-decreasing function of $k=0,1,2, \cdots, p_{k+1}-c q_{k+1}$ has at most one change of sign, and if one change occurs, it occurs from + to - . By the variation diminishing property of $Z_{k}(t)=\exp (-\lambda t)(\lambda t)^{k} / k$ !, it follows that the same statement may be made for $f(t)-c g(t)$ as a function of $t$, implying that $f(t) / g(t)$ is a non-decreasing function of $t$.

(ii) Let $\bar{P}_{k} \geqq{ }^{\mathrm{FR}} \bar{Q}_{k}$. This implies that

$$
\begin{aligned}
& \frac{\bar{P}_{k-1}-\bar{P}_{k}}{\bar{P}_{k}} \leqq \frac{\bar{Q}_{k-1}-\bar{Q}_{k}}{\bar{Q}_{k}}, \quad k=1,2, \cdots, \\
& \Rightarrow \frac{\bar{P}_{k}}{\bar{Q}_{k}} \text { is a non-decreasing function of } k=0,1,2, \cdots,
\end{aligned}
$$

Now for any real $c$,

$$
\bar{F}(t)-c \bar{G}(t)=\sum_{k=0}^{\infty} \frac{\exp (-\lambda t)(\lambda t)^{k}}{k !}\left(\bar{P}_{k}-c \bar{Q}_{k}\right) .
$$

By using similar arguments as in (i), it follows that $\bar{F}(t) / \bar{G}(t)$ is a non-decreasing function of $t$ implying that $f(t) / \bar{F}(t) \leqq g(t) \bar{G}(t) \forall t \geqq 0$ (cf. Ross (1983), p. 281).

(iii) This has a trivial proof.

(iv) It follows from the fact that for any $t \geqq 0$

$$
\left.\int_{t}^{\infty} \bar{F}(u) d u-\int_{t}^{\infty} \bar{G}(u) d u=\frac{1}{\lambda} \sum_{j=0}^{\infty} \frac{\exp (-\lambda t)(\lambda t)^{j}}{j !} \sum_{k=j}^{\infty}-\bar{P}_{k}-\bar{Q}_{k}\right) .
$$

(v) Since $\bar{P}_{k} \geqq{ }^{\mathrm{MR}} \bar{Q}_{k}$, we have

$$
\begin{aligned}
\bar{Q}_{j} & \sum_{k=j}^{\infty} \bar{P}_{k} \geqq \bar{P}_{j} \sum_{k=j}^{\infty} \bar{Q}_{k}, \quad j=0,1,2, \cdots \\
\Rightarrow & \left(\sum_{k=j}^{\infty} \bar{Q}_{k}-\sum_{k=j+1}^{\infty} \bar{Q}_{k}\right) \sum_{k=j}^{\infty} \bar{P}_{k} \geqq\left(\sum_{k=j}^{\infty} \bar{P}_{k}-\sum_{k=j+1}^{\infty} \bar{P}_{k}\right) \sum_{k=j}^{\infty} \bar{Q}_{k} \\
\Rightarrow & \frac{\sum_{k=j+1}^{\infty} \bar{P}_{k}}{\sum_{k=j+1}^{\infty} \bar{Q}_{k}} \geqq \frac{\sum_{k=j}^{\infty} \bar{P}_{k}}{\sum_{k=j}^{\infty} \bar{Q}_{k}}, \quad j=0,1,2, \ldots \\
\Rightarrow & \frac{\sum_{k=j}^{\infty} \bar{P}_{k}}{\sum_{k=j}^{\infty} \bar{Q}_{k}} \text { is a non-decreasing function of } j=0,1,2, \ldots
\end{aligned}
$$

For any real $C$, we have

$$
\int_{t}^{\infty} \bar{F}(u) d u-C \int_{t}^{\infty} \bar{G}(u) d u=\frac{1}{\lambda} \sum_{j=0}^{\infty} \frac{\exp (-\lambda t)(\lambda t)^{k}}{j !}\left\{\sum_{k=j}^{\infty} \bar{P}_{k}-C \sum_{k=j}^{\infty} \bar{Q}_{k}\right\} .
$$

By using arguments similar to (i), it follows from (2.6) that $\int_{t}^{\infty} \bar{F}(u) d u / \int_{t}^{\infty} \bar{G}(u) d u$ is a 
non-decreasing function of $t \geqq 0$ implying that

$$
\frac{1}{\bar{F}(t)} \int_{t}^{\infty} \bar{F}(u) d u \geqq \frac{1}{\bar{G}(t)} \int_{t}^{\infty} \bar{G}(u) d u, \quad \forall t \geqq 0 .
$$

See Deshpande et al. (1988).

\section{References}

Deshpande, J. V., Singh, H., Bagai, I. And Jain, K. (1988) Some partial orders describing positive ageing. To appear.

Esary, J. D., Marshall, A. W. and Proschan, F. (1973) Shock models and wear processes. Ann. Prob. 1, 627-649.

Karlin, S. (1968) Total Positivity. Vol. I. Stanford University Press, Stanford.

KLEFSJÖ, B. (1981) HNBUE survival under some shock models. Scand. J. Statist. 8, 39-47.

Ross, S. M. (1983) Stochastic Processes. Wiley, New York.

SingH, H. (1988) On partial orderings of life distributions. Naval Res. Logist. Quart. 35, 53-60. 